\title{
Transformation de la 19-hydroxyandrostènedione en œstrone et en œstradiol par le testicule embryonnaire de rat en culture in vitro
}

\author{
JP Weniger *, A Zeis \\ Université Louis-Pasteur, Laboratoire de zoologie et d'embryologie expérimentale \\ 12, rue de l'Université, 67000 Strasbourg, France
}

(Reçu le 10 juillet 1989; accepté le 17 janvier 1990)

Résumé - L'activité aromatasique du testicule embryonnaire de rat a été démontrée par la transformation de la testostérone $\left[{ }^{3} \mathrm{H}\right]$ en cestradiol. Cependant, le pourcentage de transformation était faible, de l'ordre de $0,06 \%$, vraisemblablement à cause de la forte dilution du précurseur radioactif par les grandes quantités de testostérone endogène produites. C'est pourquoi, on a essayé d'améliorer le taux de conversion en utilisant un précurseur situé en aval de la testostérone sur la voie de biosynthèse des œstrogènes. Des testicules d'embryon de rat de 18-21 jours ont été cultivés in vitro en présence de 19-hydroxyandrostènedione $\left[{ }^{3} \mathrm{H}\right]$, dont on a déterminé le taux de conversion en œstrone $\left[{ }^{3} \mathrm{H}\right]$ et en cestradiol $\left[{ }^{3} \mathrm{H}\right]$ par double dilution isotopique et recristallisation à activité spécifique constante. L'action stimulatrice de $\mathrm{LH}, \mathrm{FSH}$ et du $(\mathrm{Bu})_{2} \mathrm{AMPc}$ a été étudiée. En comparaison avec les résultats obtenus précédemment en utilisant la testostérone comme précurseur, le taux de synthèse des œestrogènes a été augmenté de 5-10 fois. FSH et le (Bu $)_{2}$ AMPc ont stimulé l'activité aromatasique, LH étant sans effet. Ces résultats apportent la preuve définitive de la capacité du testicule embryonnaire de rat à aromatiser les androgènes.

aromatase / œstrogène / œstrone / œstradiol / testicule embryonnaire

Summary - Aromatization of 19-hydroxyandrostenedione by the fcetal rat testis in vitro. Aromatase activity in the foetal rat testis was demonstrated by the conversion of $\left[{ }^{3} \mathrm{H}\right]$ testosterone into cestradiol. However, the conversion rate was low, around $0.06 \%$, probably because the radioactive precursor was heavily diluted by the large amounts of endogenous testosterone produced. In an attempt to improve the conversion rate, we therefore used a precursor following testosterone on the cestrogen biosynthesis pathway. Testes from 18-21-day-old fotal rats were cultured in vitro with $\left.{ }^{B} \mathrm{H}\right]$ 19-hydroxyandrostenedione as a substrate in the presence or absence of $\mathrm{LH}, \mathrm{FSH}$ or $(\mathrm{Bu})_{2}$ CAMP. The $\left[{ }^{3} \mathrm{H}\right]$ œstrone and $\left[{ }^{3} \mathrm{H}\right]$ œstradiol synthesized were measured by double isotopic dilution and recrystallization to constant specific activity. In comparison with previous results obtained with testosterone as precursor, the synthesis rate of cestrogens was increased 5-10-fold. FSH and $(\mathrm{Bu})_{2}$ cAMP stimulated aromatase activity; $L H$ had no effect. These results definitively establish the capacity of the fortal rat testis to aromatize androgens.

aromatase / cestrogen / cestrone / cestradiol / fotal testis

* Correspondance et tirés à part 


\section{INTRODUCTION}

Le testicule d'étalon était connu pour sa grande richesse en cestrone et en œstradiol (Beall, 1940). Après qu'on eut démontré leur sécrétion par le testicule chez de nombreuses autres espèces, $y$ compris l'homme (Baird et al, 1973), l'idée était proche de voir si elle existait déjà pendant la vie embryonnaire. C'était le cas, mais les quantités formées étaient très faibles, peut-être en raison de la forte dilution de la testostérone tritiée servant de précurseur par les grandes quantités de testostérone endogène (Weniger et Zeis, 1983). Toutefois, le taux de synthèse d'œstrogènes ne fut nullement amélioré en utilisant des précurseurs moins abondants, comme l'androstènedione ou la déhydroépiandrostérone (Weniger et Zeis, 1985, 1987). Plutôt que d'être aromatisés directement, ils étaient vraisemblablement d'abord transformés en testostérone. II convenait donc de choisir un précurseur des œestrogènes situé en aval de la testostérone sur la voie de biosynthèse, en l'occurrence la 19-hydroxyandrostènedione. L'oxydation en C-19 est en effet la première de 3 oxydations successives catalysées par l'aromatase et conduisant aux œstrogènes (Fishman et Goto, 1981; Fishman et Raju, 1981). Malgré la présence de testostérone endogène, on pouvait supposer que l'aromatase transformerait préférentiellement la 19-hydroxyandrostènedione exogène. Nous avons donc étudié la formation in vitro d'œstrone et d'œstradiol à partir de 19-hydroxyandrostènedione par le testicule embryonnaire de rat stimulée ou non par différentes substances ( $\mathrm{LH}$, $\mathrm{FSH}$ ou $\left.(\mathrm{Bu})_{2} \mathrm{AMPc}\right)$.

\section{MATÉRIEL ET MÉTHODE}

\section{Culture d'organes}

Les testicules ont été prélevés aseptiquement sur des embryons de rat Wistar de 18 à 21 jours et découpés selon leur taille en 2 à 5 fragments. Les explants découpés sur 4 à 10 testicules ont été cultivés dans une boîte Falcon sur un filtre Millipore HTAF à la surface de $100 \mu \mathrm{l}$ de milieu 199 contenant $1 \mu \mathrm{Ci}$ de $\left[6,7-{ }^{3} \mathrm{H}\right]$ 19hydroxyandrostènedione (Du Pont de Nemours, NEN Products; AS : $46 \mathrm{Ci} / \mathrm{mmol}$ ). Les boîtes Falcon ont été placées pendant $24 \mathrm{~h}$ à $37^{\circ} \mathrm{C}$ dans une enceinte d'incubation enrichie en $\mathrm{O}_{2}$ et $\mathrm{CO}_{2}(95 / 5)$. Les milieux ont ensuite été examinés attentivement pour déceler une éventuelle infection, puis congelés $\left(-20^{\circ} \mathrm{C}\right)$ jusqu'au moment de l'analyse. Dans chaque série expérimentale, 1 ou 2 explants ont été fixés pour l'étude histologique.

\section{Methode d'analyse}

Elle a déjà été publiée (Weniger et Zeis, 1987) et permet la détermination du pourcentage de transformation du précurseur radioactif en cestrone et en cestradiol. La quantité totale de radioactivité présente dans l'œstrone ou l'œestradiol, multipliée par l'inverse du pourcentage de récupération, est divisée par l'activité mise en jeu.

\section{Substances testées}

La LH bovine (NIH-LH-B9) et la FSH ovine (NIADDK-o FSH-17) étaient un don du NIH; le dibutyryl AMPc provenait de Sigma. FSH était utilisée aux concentrations courantes de 0,1 aे 5 $\mu \mathrm{g} / \mathrm{ml}$, LH à la concentration de $5 \mu \mathrm{g} / \mathrm{ml}$ et le (Bu) ${ }_{2}$ AMPc à la concentration de $0,5 \mathrm{mmol}$, où ses effets sont réputés maximum (George et Ojeda, 1987). 


\section{RÉSULTATS}

Les explants étaient en bonne condition, comme l'attestait l'observation de figures de mitose de cellules intra- aussi bien qu'intertubulaires. Le tableau I donne les résultats de l'ensemble des expériences. On peut faire les constatations suivantes.

Le taux de synthèse d'œstrogènes est augmenté en présence de $(\mathrm{Bu})_{2} \mathrm{AMPc}$. L'action de FSH est nette dans tous les cas à la concentration de $5 \mu \mathrm{g} / \mathrm{ml}$. Elle semble nulle à la concentration de $0,1 \mu \mathrm{g} /$ $\mathrm{ml}$ et faible à celle de $0,5 \mu \mathrm{g} / \mathrm{ml}$. Même à la concentration de $5 \mu \mathrm{g} / \mathrm{ml}$, qui est forte, $\mathrm{LH}$ est sans action. Bien que le produit d'aro- matisation direct de la 19-hydroxyandrostènedione soit l'œstrone, c'est l'œstradiol qui est formé préférentiellement.

\section{DISCUSSION}

En utilisant la 19-hydroxyandrostènedione comme précurseur des œstrogènes formés par le testicule embryonnaire de rat, nous avons obtenu des taux de synthèse 5 à 10 fois plus importants qu'en partant de testostérone (Weniger et Zeis, 1988). La stimulation de la synthèse des œstrogènes par FSH est confirmée. Elle s'explique par la présence de récepteurs de FSH dans le

Tableau I. Transformation de la 19-hydroxyandrostènedione $\left[{ }^{3} \mathrm{H}\right]$ en œstrone $\left(E_{1}\right)$ et en œstradiol $\left(E_{2}\right)$ par le testicule embryonnaire de rat. Les résultats sont exprimés en taux de conversion par expérience (mesures individuelles pour chacune d'entre elles).

Expériences

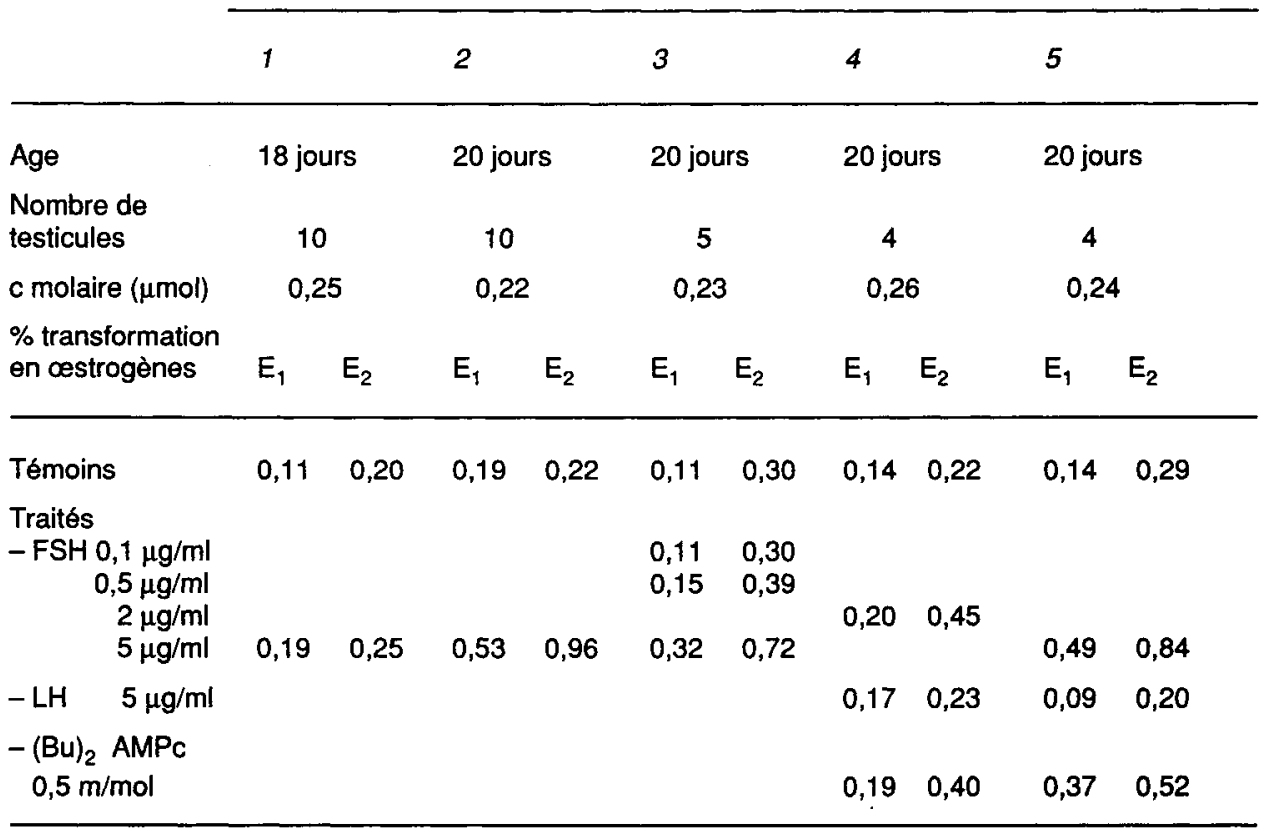


testicule embryonnaire de rat dès le stade de 17 jours (Warren et al, 1984). Le fait que l'œstradiol soit prédominant, bien que le produit d'aromatisation direct de la 19hydroxyandrostènedione soit l'œstrone, révèle la présence d'une 17-oxydoréductase active dans le sens de la réduction, donc stimulée elle aussi par FSH.

L'absence d'action de $\mathrm{LH}$ mérite un commentaire. En présence de LH, l'activité aromatasique de cultures de cellules de Leydig provenant de testicules de ratons âgés de 20 jours est augmentée de $180 \%$, FSH étant sans effet (Papadopoulos et al, 1986). L'activité aromatasique de cellules de Leydig provenant de testicules embryonnaires de 21 jours et cultivées pendant 3 jours n'était plus augmentée que de $30 \%$ (Tsai-Morris et al, 1986). En extrapolant ces résultats aux stades de 18-20 jours de gestation, on s'attend à ce que LH soit sans action. Comme d'autre part la stimulation de l'activité aromatasique de cultures de cellules de Sertoli provenant de testicules de jeunes ratons est d'autant plus intense que les testicules sont jeunes (Papadopoulos et al, 1986), on peut supposer que l'activité aromatasique des testicules embryonnaires soit le propre des cellules de Sertoli.

On ignore si les œstrogènes jouent un rôle dans le développement et le fonctionnement du testicule embryonnaire. On ignore aussi si FSH stimule l'activité aromatasique du testicule in vivo. Notons cependant que des cellules élaboratrices de FSH ont été décelées par immunocytochimie dans l'hypophyse antérieure d'embryons de rat de 19 jours (Tougard et al, 1977; Watanabe et Daikoku, 1979) et que le dosage radioimmunologique de $\mathrm{FSH}$ dans l'hypophyse d'embryons mâles a donné des résultats positifs dès le stade de 17 jours (Chowdhury et Steinberger, 1976).
En conclusion, le présent travail démontre l'existence d'une activité aromatasique, ainsi que d'une activité $17 \beta$ hydroxystéroïde-déshydrogénasique dans le testicule embryonnaire de rat de 18-20 jours, toutes deux stimulables par FSH.

\section{RÉFÉRENCES}

Beall D (1940) Isolation of $\alpha$-œstradiol and œstrone from horse testes. Biochem J 34, 1293-1298

Baird DT, Galbraith A, Fraser IS, Newsam JE (1973) The concentration of cestrone and cestradiol-17 $\beta$ in spermatic venous blood in man. J Endocrino/ 57, 285-288

Chowdhury M, Steinberger E (1976) Pituitary and plasma levels of gonadotropins in fœtal and newborn male and female rats. $J$ Endocrinol 69, 381-384

Fishman J, Goto J (1981) Mechanism of estrogen biosynthesis. Participation of multiple enzyme sites in placental aromatase hydroxylations. J Biol Chem 256, 4466-4471

Fishman J, Raju MS (1981) Mechanisms of estrogen biosynthesis. Stereochemistry of $\mathrm{C}-1$ hydrogen elimination in the aromatization of $2 \beta$-hydroxy-19-oxoandrostenedione. J Biol Chem 256, 4472-4477

George FW, Ojeda SR (1987) Vasoactive intestinal peptide enhances aromatase activity in the neonatal rat ovary before development of primary follicles or responsiveness to follicle - stimulating hormone. Proc Natl Acad Sci USA 84, 5803-5807

Papadopoulos V, Carreau S, Szerman-Joly E, Drosdowsky MA (1986) Rat testis 17 œstradiol : identification by gas chromatography mass-spectrometry and age related cellular distribution. J Steroid Biochem 24, 1211-1216

Tougard C, Picart A, Tixier-Vidal A (1977) Cytogenesis of immunoreactive gonadotropic cells in the fœtal rat pituitary at light and electron microscope levels. Dev Bio/ 58, 148-163

Tsai-Morris CH, Knox G, Luna S, Dufau ML (1986) Acquisition of cestradiol-mediated regulatory mechanism of steroidogenesis in cultured fœtal rat Leydig cells. $J$ Biol Chem $261,3471-3474$ 
Warren DW, Huhtaniemi IT, Tapanainen J, Dufau ML, Catt KJ (1984) Ontogeny of gonadotropin receptors in the fotal and neonatal rat testis. Endocrinology 114, 470-476

Watanabe YG, Daikoku S (1979) An immunohistochemical study of the cytogenesis of adenohypophysial cells in fœtal rats. Dev Biol $68,557-567$

Weniger JP, Zeis A (1983) Aromatisation de la testostérone par le testicule embryonnaire de rat. CR Acad Sci Ser III Sci Vie 296, 293-296
Weniger JP, Zeis A (1985) Comparaison du métabolisme des stéroïdes par les testicules et les ovaires de l'embryon de rat de 19 jours en culture in vitro. Reprod Nutr Dév 25, 531535

Weniger JP, Zeis A (1987) CEstrogen synthesis by the fotal rat testis in organ culture. J Steroid Biochem 28, 307-310

Weniger JP, Zeis A (1988) Stimulation of aromatase activity in the fœtal rat gonads by cAMP and FSH. Acta Endocrinol 119, 381-385 